\title{
Comparación del plan agregado de producción bajo metodologías de autores americanos y europeos
} Comparison of the aggregate production plan under methodologies of American and European authors

Felipe Chaves-Aguilar ${ }^{1}$, Juan Carlos Ocampo-Rodríguez ${ }^{2}$, Pamela Araya-Martínez ${ }^{3}$, Marcela Meneses-Guzmán ${ }^{4}$, Rafael Gutiérrez-Brenes ${ }^{5}$

Chaves-Aguilar, F; Ocampo-Rodríguez, J; Araya-Martínez, P; Meneses-Guzmán, M; Gutiérrez-Brenes, R. Comparación del plan agregado de producción bajo metodologías de autores americanos y europeos. Tecnología en Marcha. Vol. 33-2. Abril-Junio 2020. Pág 17-26.

doi) https://doi.org/10.18845/tm.v33i2.4201

Fecha de recepción: 16 de abril de 2019 Fecha de aprobación: 30 de julio de 2019

1 Estudiante. Licenciatura en Ingeniería en Producción Industrial. Instituto Tecnológico de Costa Rica. Costa Rica. Correo electrónico: fechagui13@hotmail.com.

2 Estudiante. Licenciatura en Ingeniería en Producción Industrial. Instituto Tecnológico de Costa Rica. Costa Rica. Correo electrónico: juancarlosocampo2@gmail.com

3 Estudiante. Licenciatura en Ingeniería en Producción Industrial. Instituto Tecnológico de Costa Rica. Costa Rica. Correo electrónico: pamelaam21@gmail.com.

4 Profesora e investigadora. Escuela de Producción Industrial. Instituto Tecnológico de Costa Rica. Costa Rica. Correo electrónico: mameneses@tec.ac.cr.

5 Profesor e investigador. Escuela de Producción Industrial. Instituto Tecnológico de Costa Rica. Costa Rica. Correo electrónico: guti@tec.ac.cr. 


\title{
Palabras clave
}

Plan agregado de producción; Control de producción; Costo de producción; Fuerza laboral.

\section{Resumen}

El objetivo del estudio fue comparar el costo del plan agregado de fabricación de botellas de tereftalato de polietileno (PET) calculado mediante metodologías americana y europea para seis escenarios del plan agregado del proceso, cuya diferencia radica en la modificación de sus variables de decisión. Los pronósticos de la demanda se estimaron mediante el método Winters, los resultados de los planes agregados en la aplicación del caso de estudio se obtuvieron mediante la aplicación del método de la transformada inversa para una distribución uniforme. Se observa que el costo de producción del plan agregado por metodologías de autores europeos es mayor debido a que considera más variables como mano de obra y órdenes atrasadas que el enfoque americano no suma. Sin embargo, no se puede tomar una decisión si existen más o menos variables de costo ya que son los pronósticos los que dictan el rumbo.

\section{Keywords}

Aggregate production plan; Production control; Production cost; Labor force.

\begin{abstract}
The objective of the study was to compare the cost of the aggregate polyethylene terephthalate (PET) bottle manufacturing plan calculated by American and European methodologies for six scenarios of the aggregate process plan, whose difference lies in the modification of its decision variables. Demand forecasts were estimated using the Winters method, the results of the aggregate plans in the application of the case study were based on the application of the inverse transform method for a uniform distribution. It is observed that the cost of production of the aggregate plan by methodologies of European authors is greater because it considers more variables such as labor and backlogs that the American approach does not add up. Normally you cannot make a decision if there are more or fewer variables of cost since it is the forecasts that dictate the course.
\end{abstract}

\section{Introducción}

Control de producción es planear, coordinar, ejecutar y controlar los recursos productivos para realizar los planes establecidos a nivel estratégico, operativo y táctico [1] [2] [3]; a nivel estratégico contribuye a establecer los objetivos y las metas de las organizaciones [4] [5] a nivel más operativo, contribuye con la planificación de los recursos empresariales y con una de las partes más sensibles de la administración de una organización como es el manejo de pronósticos y sus costos asociados [6], mientras que a nivel táctico se controla en la producción.

Sipper [2] señala que existen dos metodologías utilizadas para llevar a cabo el control de producción, la primera llamada europea e impulsada por Domínguez Machuca y la segunda llamada americana e implementada por Steven Nahmias y Thomas Vollmann. Ambos enfoques se dividen en tres etapas: plan agregado (PA), plan maestro de producción (PMP) y plan de requerimiento de materiales (MRP) [7]. Teniendo en cuenta que la planificación es el paso previo a la ejecución y control de la producción [8], la planeación agregada se encarga del desarrollo del presupuesto de operación y determina los niveles de fuerza laboral e inventario [9]. 
La planeación agregada desde sus inicios ha otorgado gran ventaja para obtener el análisis de las necesidades de insumos para fabricar y distribuir porque propicia conocer la demanda en un horizonte de tiempo, permitiendo que las organizaciones trabajen a pleno en sus tiempos de producción y despacho de producto terminado, para que su rendimiento y costos se vean reflejados positivamente [10]. A mediados de los años 50 la planeación agregada fue formalizada por Holt, Modigliani, Simon y Muth [11] [12] [13], siendo aplicada en materia de dirección de operaciones [14] [15]. En las últimas décadas, las estrategias para llevar a cabo la planificación agregada han cambiado constantemente [16] generándose principalmente dos corrientes formales de pensamiento: en España impulsada por Machuca en la década de los noventa y en Estados Unidos por Vollmann y Nahmias a inicios de los años 2000. Se identifican otros esfuerzos diferentes de autores provenientes de India, Malasia, México y Brasil [16] [17] [18].

Considerando que ambos enfoques, el americano y europeo, utilizan la planeación agregada para ajustar la capacidad de producción a la demanda de una organización y muestra el costo total de producción [8], el objetivo de este estudio consiste en comparar el manejo de los costos del plan agregado de producción propuesto por cada enfoque.

Entre los insumos para elaborar el plan agregado se encuentran: pronósticos, restricciones, costos, variables de decisión y criterios de evaluación [10] [19] [20].

El objetivo de los pronósticos es revelar previsiones en un tiempo adecuado y contemplando un nivel alto de exactitud [2] [21] [22]. Para ejecutar la planeación agregada se traducen los pronósticos de la demanda en un esquema de planeación que toma en cuenta la fuerza laboral y la producción durante un periodo determinado [23].

Ambos enfoques utilizan métodos de programación lineal para desarrollar el plan agregado el cual es uno de los más importantes y difundidos en la literatura para este objetivo [10] [24]. Adicionalmente, Machuca, desarrolla la planeación agregada transformando los pronósticos en unidades agregadas, donde satisface planes a largo plazo con el menor costo posible.

Los costos empleados por la metodología de autores europeos contemplan los costos de producción (mano de obra y materiales), inventario (almacenaje y faltantes) y capacidad (contratación y capacitación) mientras que la de autores americanos contemplan costos de suavización, faltantes, tiempos normales, tiempos extras y tiempos muertos, costos de despido, contrataciones y mantener unidad en inventario.

Las variables de decisión para ambos enfoques son inventario, nivel de fuerza laboral, nivel de producción, horizonte de planeación [10]. Cabe mencionar que las metodologías de autores americanos y europeos no consideran las restricciones ni criterios de evaluación.

Los planes agregados se pueden llevar a cabo por medio de planes cero inventarios para movilizar la fuerza laboral con la intención de tener un nivel de inventario mínimo almacenado [23] o planes de trabajo de fuerza constante donde se elimina por completo la necesidad de contratar o despedir durante el horizonte de planeación, teniendo la cantidad óptima de fuerza laboral [1] [23].

Para establecer una mejor asignación y ordenamiento de los recursos en el tiempo, se realiza el plan maestro de producción que utiliza la información que proporciona el plan agregado [19] [25]. La idea principal del plan maestro de producción es traducir la información que brinda el plan agregado a unidades específicas, considerando como base los pronósticos obtenidos en este plan. El plan de requerimiento de materiales (MRP) se encarga de transformar, el plan maestro de producción en un programa que detalla las necesidades de materiales y componentes para la fabricación del producto final [19] [26]. El MRP debe determinar el tiempo de producción que considere la cantidad de materiales y componentes necesarios para la 
producción [1]. De aquí el impacto que posee el plan agregado de producción en las demás etapas para llevar a cabo el control de producción.

El trabajo se encuentra organizado de la siguiente forma, primero se exponen los modelos empleados por ambos enfoques para determinar el costo de producción. Seguidamente se presenta el caso de estudio y se presentan los enunciados que modifican las variables de decisión. En la sección de resultados se presenta los pronósticos estimados de la demanda y los costos del plan agregado tanto de la aplicación como de los enunciados para cada enfoque. La discusión se realiza comparando los resultados para el plan cero inventarios y para el plan fuerza constante. Al final se presentan las principales conclusiones.

\section{Metodologías para generar el plan agregado}

Las variables y costos utilizados por metodologías americana y europea para determinar plan agregado se muestran en cuadro 1; ambas metodologías consideran las mismas variables, excepto que el enfoque europeo, adicionalmente, considera la cantidad de mano de obra y cantidad y costo de órdenes atrasadas.

Cuadro 1. Parámetros utilizados en el plan agregado por ambas metodologías

\begin{tabular}{|c|c|c|c|c|}
\hline \multirow[b]{3}{*}{ Plan } & \multicolumn{4}{|c|}{ Metodología de autores } \\
\hline & \multicolumn{2}{|c|}{ Americanos } & \multicolumn{2}{|c|}{ Europeos } \\
\hline & Cero inventarios & Fuerza constante & Cero inventarios & Fuerza constante \\
\hline \multirow{10}{*}{ Variables } & $\mathrm{n}_{\mathrm{t}}$ : Periodo de producción & $\mathrm{n}_{\mathrm{t}}$ : Periodo de producción & $\mathrm{n}_{\mathrm{t}}:$ Periodo de producción & $\mathrm{n}_{\mathrm{t}}:$ Periodo de producción \\
\hline & $\mathbf{k}_{\mathbf{t}}$ : Unidades por trabajador & $\mathbf{k}_{\mathbf{t}}$ : Unidades por trabajador & $\mathrm{k}_{\mathrm{t}}$ : Unidades por trabajador & $\mathrm{k}_{\mathrm{t}}$ : Unidades por trabajador \\
\hline & $\mathrm{K}_{\mathrm{t}}$ : Unidades Producidas & $\mathrm{K}_{\mathrm{t}}$ : Unidades Producidas & $\mathrm{K}_{\mathrm{t}}$ : Unidades Producidas & $\mathbf{K}_{\mathbf{t}}$ : Unidades Producidas \\
\hline & $\mathrm{D}_{\mathrm{t}}:$ Pronósticos & $\mathrm{D}_{\mathrm{t}}$ : Pronósticos & $\mathrm{D}_{\mathrm{t}}$ : Pronósticos & $\mathrm{D}_{\mathrm{t}}$ : Pronósticos \\
\hline & $\mathrm{W}_{\mathrm{t}}$ : Nivel de fuerza laboral & $\mathrm{Wm}_{\mathrm{t}}$ : Nivel de fuerza laboral mayor & $\mathrm{W}_{\mathrm{t}}$ : Nivel de fuerza laboral & $\mathrm{Wm}_{\mathrm{t}}$ : Nivel de fuerza laboral mayor \\
\hline & $\mathbf{I}_{\mathbf{t}}:$ Nivel de inventario & $\mathrm{I}_{\mathrm{t}}$ : Nivel de inventario & $\mathbf{I}_{\mathbf{t}}:$ Nivel de inventario & $\mathbf{I}_{\mathbf{t}}:$ Nivel de inventario \\
\hline & $\mathbf{P}_{\mathbf{t}}$ : Nivel de producción & $\mathbf{P}_{\mathbf{t}}$ : Nivel de producción & $\mathbf{P}_{\mathbf{t}}$ : Nivel de producción & $\mathbf{P}_{\mathbf{t}} \quad:$ Nivel de producción \\
\hline & $\mathrm{H}_{\mathrm{t}}$ : Cantidad de contrataciones & $\mathrm{H}_{\mathrm{t}}$ : Cantidad de contrataciones & $\mathrm{H}_{\mathrm{t}}$ : Cantidad de contrataciones & $\mathrm{H}_{\mathrm{t}}$ : Cantidad de contrataciones \\
\hline & $\mathbf{F}_{\mathbf{t}}$ : Cantidad de despidos & $\mathbf{F}_{\mathbf{t}}$ : Cantidad de despidos & $\mathbf{F}_{\mathbf{t}}:$ Cantidad de despidos & $\mathbf{F}_{\mathbf{t}}$ : Cantidad de despidos \\
\hline & & & $\mathrm{O}_{\mathrm{t}}$ : Cantidad de órdenes atrasadas & $\mathrm{O}_{\mathrm{t}}$ : Cantidad de órdenes atrasadas \\
\hline \multirow{5}{*}{ Costos } & $\mathrm{C}_{\mathrm{H}}$ : Contrataciones & & $\mathrm{C}_{\mathrm{H}}$ : Contrataciones & \\
\hline & $\mathrm{C}_{\mathrm{F}}:$ Despidos & & $\mathrm{C}_{\mathrm{F}}$ : Despidos & \\
\hline & $\mathrm{C}_{\mathrm{I}}$ : Unidad en inventario & & $\mathrm{C}_{\mathrm{I}}$ : Unidad en inventario & \\
\hline & & & $\mathrm{C}_{\mathrm{M}}$ : Mano de obra & \\
\hline & & & $\mathrm{C}_{\mathrm{o}}$ : Órdenes atrasadas & \\
\hline
\end{tabular}

Parámetros de modelo

$$
\begin{gathered}
K_{t}=n_{t} * k_{t} \\
W_{t}=D_{t} / K_{t} \text { ó } W_{m t}=\max \left\{D_{t} / K_{t}\right\} \\
P_{t}=K_{t} * W_{t} \text { ó } P_{t}=K_{t} * W_{m t} \\
I_{t}=D_{t}-P_{t}
\end{gathered}
$$

Costo de producción (CP) del plan agregado cero inventarios y fuerza constante de autores americanos

$$
C P=\sum_{t=1}^{T}\left(C_{H} * H_{t}+C_{F} * F_{t}+C_{I} * I_{t}\right)
$$


Costo de producción (CP) del plan agregado cero inventarios

y fuerza constante de autores europeos

$$
C P=\sum_{t=1}^{T}\left(C_{H} * H_{t}+C_{F} * F_{t}+C_{M} * W_{t}+C_{I} * I_{t}+C_{O} * O_{t}\right)
$$

\section{Aplicación en un caso de estudio}

El caso de estudio es en una empresa dedicada a la fabricación de botellas de tereftalato de polietileno (PET), en dos líneas de producción en el periodo de octubre 2016 a setiembre 2017. La empresa cuenta con 6 trabajadores para la fabricación, el horizonte de producción es de 26 días y cuenta con una razón de producción de 100.000 botellas por día de tres tipos de botellas. Los valores observados de la demanda en un periodo de tiempo $\left(\mathrm{X}_{t}\right)$ fueron obtenidos del informe de producción brindado por el departamento de operaciones. El estudio contempla el impacto del plan agregado de producción para toda la producción

\section{Pronósticos}

Se utiliza el método suavizado exponencial de Winters ya que los datos son aleatorios, con tendencia y estacionalidad [2], donde el pronóstico para el periodo $t\left(D_{t}\right)$ se determina por medio de la ecuación 3 utilizando un error medio de 0,05.

Donde:

$$
D_{t}=\left(S_{t}+k B_{t}\right) * C_{t+k-g l}
$$

$$
\begin{gathered}
S_{t}=\alpha\left(\frac{X_{t}}{C_{t-L}}\right)+(1-\alpha)\left(S_{t-1}+B_{t-1}\right) \\
B_{t}=\beta\left(S_{t}-S_{t-1}\right)+(1-\beta) B_{t-1} \\
S_{t}=\gamma\left(\frac{X_{t}}{S_{t}}\right)+(1-\gamma) C_{t-1} \\
\alpha=\left(\frac{2}{n+1}\right) \\
\beta \leq\left(\frac{2}{\alpha}-1\right)=\sqrt{\left(\frac{2}{\alpha}-1\right)^{2}-1} \\
\gamma=0,05
\end{gathered}
$$

$X_{t}$ : valor observado en el periodo $t$

$\mathrm{S}_{\mathrm{t}}$ : valor estimado aleatorio en periodo $\mathrm{t}$

$\mathrm{B}_{\mathrm{t}}$ : valor estimado tendencia en periodo $\mathrm{t}$

$\mathrm{C}_{\mathrm{t}}$ : valor estimado estacional en periodo $\mathrm{t}$

k: periodos futuros a pronosticar

L: número de estaciones

t: periodos con datos futuros

g: entero más pequeño mayor/igual que k/L

$\gamma$ : error medio 


\section{Definición de escenarios}

Se utiliza el método de la transformada inversa de la distribución uniforme para generar 6 escenarios cada uno de los cuales presenta un escenario de aumento y disminución en cada una de las variables de decisión actuales: nivel de fuerza laboral (6 trabajadores), nivel de producción (100.000), horizonte de planeación (26 días).

Los escenarios son los siguientes:

Escenario 1. Días entre 26-30.

Escenario 2. Días entre 20-26.

Escenario 3. Fuerza laboral de 0 a 6 colaboradores.

Escenario 4. Fuerza laboral de 6 a 10 colaboradores.

Escenario 5. Nivel de producción entre 100.000 y 150.000 .

Escenario 6. Nivel de producción entre 50.000 y 100.000.

La figura 1 muestra una esquematización de la forma en que se abordarán los escenarios para cada tipo de metodología y plan.

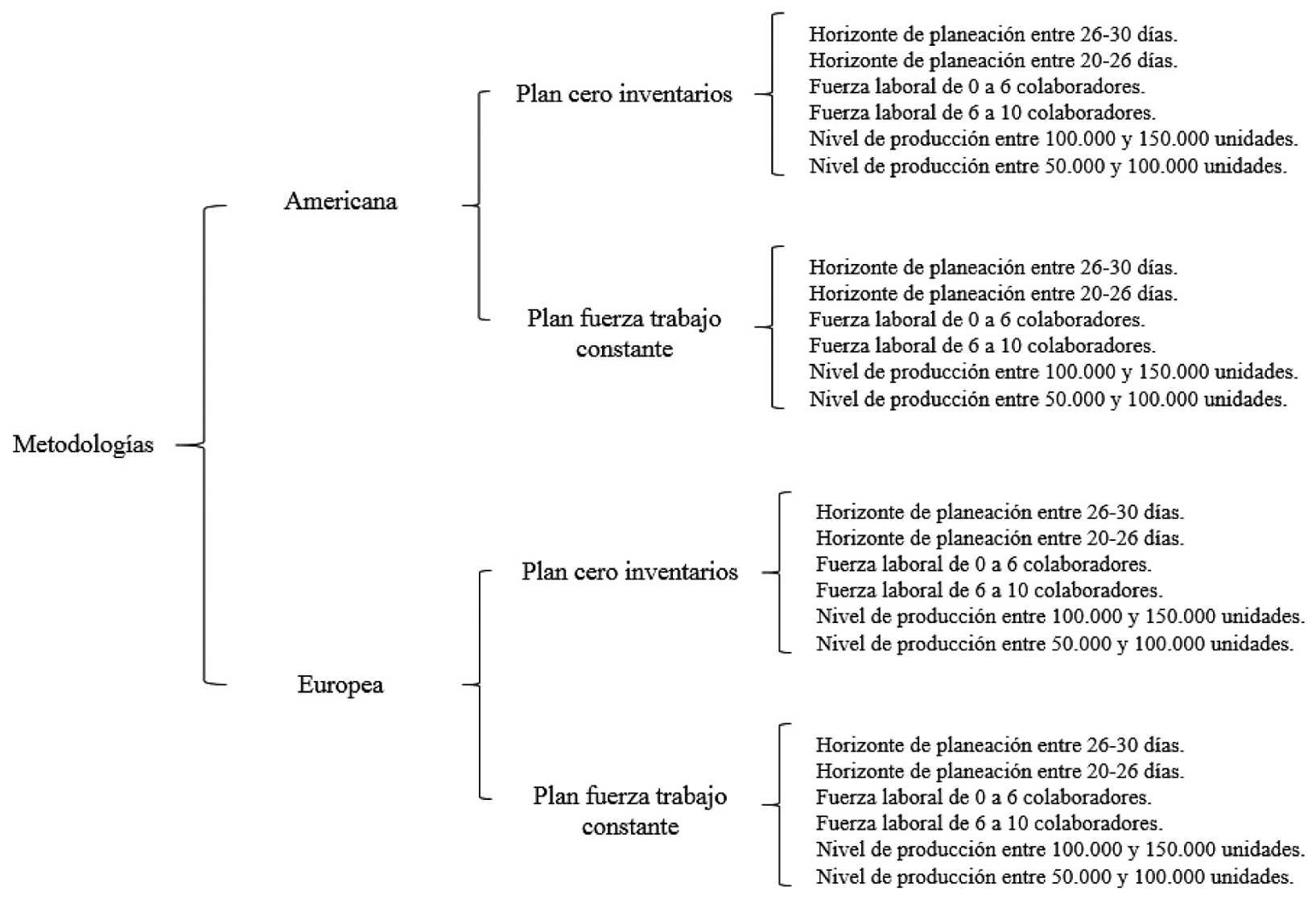

Figura 1. Aplicación de los escenarios para cada metodología y plan. 


\section{Resultados}

El cuadro 2 muestra los pronósticos, calculados mediante la ecuación (3) para cada período. Estos se mantienen constantes para el desarrollo de todos los escenarios.

Cuadro 2. Pronósticos proyectados

\begin{tabular}{lcccccc} 
Período & Enero & Febrero & Marzo & Abril & Mayo & Junio \\
\hline Pronóstico (unidades) & 991.377 & 1.081 .944 & 664.454 & 314.303 & 502.745 & 1.036 .597 \\
& & & & & & \\
Período & Julio & Agosto & Septiembre & Octubre & Noviembre & Diciembre \\
\hline Pronóstico (unidades) & 793.292 & 291.413 & 451.413 & 235.850 & 820.314 & 1.010 .911
\end{tabular}

Adicionalmente se obtuvieron los siguientes resultados un MAPE (Error Porcentual Absoluto Medio) de $0.201 \%$ el cual muestra un buen ajuste.

El cuadro 3 presenta, a partir de las ecuaciones (1) y (2), los resultados del costo total de producción y cantidad de inventario para cada uno de los planes de cero inventario y fuerza constante bajo ambas metodologías.

Cuadro 3. Costo total de producción del plan agregado

\begin{tabular}{lcccc} 
& \multicolumn{2}{c}{ Americano } & \multicolumn{3}{c}{ Europeo } \\
\cline { 2 - 5 } & Cero Inventario & Fuerza constante & Cero Inventario & Fuerza constante \\
\cline { 2 - 5 } Costo (en colones) & 23.890 .845 & 35.681 .303 & 46.011 .922 & 66.289 .947 \\
Inventario (unidades) & 605.387 & 5.005 .387 & 605.387 & 5.005 .387
\end{tabular}

Se efectuaron 250 réplicas para cada uno de los escenarios, el costo promedio de producción se muestra en el cuadro 4, mientras la cantidad de inventario promedio se muestra en el cuadro 5.

Cuadro 4. Costo total de producción del plan agregado bajo cada escenario

\begin{tabular}{|c|c|c|c|c|c|c|c|c|c|}
\hline \multirow{3}{*}{\multicolumn{2}{|c|}{ Escenario }} & \multicolumn{4}{|c|}{ Costo de producción (en colones) } & \multicolumn{4}{|c|}{ Costo de producción $(\%)$} \\
\hline & & \multicolumn{2}{|c|}{ Cero Inventario } & \multicolumn{2}{|c|}{ Fuerza constante } & \multicolumn{2}{|c|}{ Cero Inventario } & \multicolumn{2}{|c|}{ Fuerza constante } \\
\hline & & Americano & Europeo & Americano & Europeo & Americano & Europeo & Americano & Europeo \\
\hline & 1 & 24.186 .113 & 42.091 .306 & 31.951 .050 & 67.519 .794 & $1,24 \%$ & $-8,52 \%$ & $-10,45 \%$ & $1,86 \%$ \\
\hline & 2 & 28.861 .679 & 49.886 .131 & 31.781 .250 & 74.545 .005 & $20,81 \%$ & $8,42 \%$ & $-10,93 \%$ & $12,45 \%$ \\
\hline & 3 & 25.928 .815 & 47.979 .528 & 34.209 .565 & 68.312 .281 & $8,53 \%$ & $4,28 \%$ & $-4,12 \%$ & $3,05 \%$ \\
\hline & 4 & 21.938 .876 & 44.059 .953 & 30.305 .628 & 64.343 .190 & $-8,17 \%$ & $-4,24 \%$ & $-15,07 \%$ & $-2,94 \%$ \\
\hline & 5 & 22.282 .823 & 38.651 .515 & 31.037 .479 & 62.971 .778 & $-6,73 \%$ & $-16,00 \%$ & $-13,01 \%$ & $-5,01 \%$ \\
\hline & 6 & 76.775 .649 & 119.282 .569 & 269.887 .217 & 458.961 .935 & $221,36 \%$ & $159,24 \%$ & $656,38 \%$ & $592,36 \%$ \\
\hline
\end{tabular}

Los porcentajes corresponden al aumento o decremento del costo de producción de cada escenario en comparación al plan original 
Cuadro 5. Cantidad de inventario bajo cada escenario

\begin{tabular}{|c|c|c|c|c|c|c|c|c|}
\hline \multirow[b]{3}{*}{ Escenario } & \multicolumn{4}{|c|}{ Inventario (unidades) } & \multicolumn{4}{|c|}{ Inventario (\%) } \\
\hline & \multicolumn{2}{|c|}{ Cero Inventario } & \multicolumn{2}{|c|}{ Fuerza constante } & \multicolumn{2}{|c|}{ Cero Inventario } & \multicolumn{2}{|c|}{ Fuerza constante } \\
\hline & Americano & Europeo & Americano & Europeo & Americano & Europeo & Americano & Europeo \\
\hline & 683.464 & 690.956 & 5.017 .049 & 5.669 .833 & $12,90 \%$ & $14,13 \%$ & $0,23 \%$ & $13,27 \%$ \\
\hline & 515.249 & 507.279 & 4.804 .279 & 5.519 .925 & $-14,89 \%$ & $-16,21 \%$ & $-4,02 \%$ & $10,28 \%$ \\
\hline & 605.387 & 605.387 & 5.005 .387 & 5.005 .387 & $0,00 \%$ & $0,00 \%$ & $0,00 \%$ & $0,00 \%$ \\
\hline & 605.387 & 605.387 & 5.005 .387 & 5.005 .387 & $0,00 \%$ & $0,00 \%$ & $0,00 \%$ & $0,00 \%$ \\
\hline & 747.587 & 750.681 & 5.041 .840 & 5.533 .973 & $23,49 \%$ & $24,00 \%$ & $0,73 \%$ & $10,56 \%$ \\
\hline & 427.958 & 425.788 & 428.783 & 426.231 & $-29,31 \%$ & $-29,67 \%$ & $-91,43 \%$ & $-91,48 \%$ \\
\hline
\end{tabular}

Los porcentajes corresponden al aumento o decremento del inventario de cada escenario en comparación al inventario del plan original

\section{Discusión}

En general y para todos los escenarios la metodología de autores americanos presenta costos de producción menores en comparación a la metodología empleada por autores europeos. El enfoque de autores europeos contempla costos de mano de obra y de órdenes atrasadas que en la metodología de autores americanos no considera.

\section{Plan cero inventario}

Para la metodología de autores americanos en el escenario 1 el costo de producción aumenta en un 1,24\% respecto al plan original, ya que al aumentar el horizonte de planeación se crea mayor producción, consecuentemente el inventario que existe crece un 12,90\% incrementando el costo de almacenaje. En la metodología de autores europeos el costo de producción disminuye en 8,52\% al ocupar menor fuerza laboral, por su parte el inventario aumenta en un $14,13 \%$ por mantener una producción constante mayor a lo pronosticado. En el escenario 2 para ambas metodologías, al disminuir el horizonte de planeación, el inventario decrece $(14,89 \%$ en el enfoque americano y $16,21 \%$ para el enfoque europeo) ya que la producción se ajusta a los pronósticos y la fuerza laboral aumenta consecuencia de que hay menor tiempo para cumplir con la producción y un aumento en los costos de contratación y despidos.

Para el escenario 3 en ambos enfoques, al contar con menor fuerza laboral inicial el costo de producción incrementa en un 8,53\% bajo la metodología de autores americanos y 4,28\% bajo la metodología de autores europeos, esto por el aumento de contrataciones; sin embargo, el escenario 4 para ambos enfoques presenta una disminución en el costo de producción en un $8,17 \%$ y $4,24 \%$ respectivamente, ya que al contar con mayor fuerza laboral inicial se incurre en menos contrataciones. Al variar solo fuerza laboral inicial los inventarios se mantienen igual debido a que la razón de producción y el horizonte de planeación no varían.

En el escenario 5 para ambos enfoques disminuye el costo de producción en un 6,73\% para el enfoque de autores americanos y 16,00\% en el enfoque de autores europeos, esto al ser la fuerza laboral necesaria mínima por el aumento de la razón de producción, así los costos de contratación y despidos son mínimos; sin embargo, respectivamente sus inventarios aumentan en un $23.49 \%$ y un $24,00 \%$, por el aumento de la producción. Aumenta el costo de producción para ambas metodologías en el escenario 6 en un 221,36\% para el enfoque de autores americanos y $159,24 \%$ en el enfoque de autores europeos dado que la fuerza laboral debe de incrementarse para satisfacer la demanda, así aumentan los costos de contratación y despidos, pero para este escenario el inventario disminuye para ambos enfoques debido al ajuste de la producción a lo pronosticado. 


\section{Plan fuerza constante}

En la metodología de autores americanos en el escenario 1 y 2 el costo de producción disminuye, debido al decremento del costo de despidos, sin embargo, al contar con un mayor horizonte de planeación el inventario aumenta en un 0,23\% dado que la razón de producción no varía significativamente y disminuye un 4,02\% al contar con un menor horizonte de planeación. Para la metodología de autores europeos en ambos escenarios, el costo de producción aumenta, así como las unidades en inventario, ya que la razón de producción no presenta una variación significativa y el aumento del costo se debe al contemplar el costo de mano de obra.

El escenario 3 y 4 bajo la metodología de autores americanos el costo de producción disminuye debido al requerimiento de la fuerza laboral que afecta los costos de contratación y despidos, por el mismo motivo el escenario 4 bajo la metodología de autores europeos el costo disminuye, mientras que bajo este enfoque en el escenario 3 se da un aumento del costo debido al requerimiento de la fuerza laboral que aumenta el costo de mano de obra.

En el escenario 5 para ambos enfoques disminuye el costo de producción esto porque la fuerza laboral necesaria al ser mínima por el aumento de la razón de producción, los costos de contratación y despidos son menores, por el mismo motivo del plan cero inventarios la cantidad de unidades en inventario aumentan para cada enfoque. Aumenta el costo de producción para ambas metodologías en el escenario 6 en un 656,38\% para el enfoque americano y 592,36\% en el enfoque de autores europeos dado que la fuerza laboral debe de incrementarse para satisfacer la demanda, así aumentan los costos de contratación y despidos, pero para este escenario el inventario disminuye para ambos enfoques debido al ajuste de la producción a lo pronosticado.

\section{Conclusiones}

Se han comparado los costos del plan agregado de producción impulsados por autores americanos y europeos. Se han aplicado a seis escenarios en donde se varía el nivel de fuerza laboral, nivel de producción, horizonte de planeación e inventario, en la fabricación de botellas de tereftalato de polietileno (PET).

Ambas metodologías presentan similitudes en sus consideraciones para determinar el costo de producción en el plan agregado, sin embargo, la metodología europea presenta un mayor costo de producción debido a que considera más variables de costo en su determinación que el enfoque americano, como mano de obra y órdenes atrasadas.

Para ambas metodologías el resultado de los costos funciona como una guía para la toma de decisiones, pero normalmente no se puede tomar una decisión si existen más o menos variables de costo ya que son los pronósticos los que dictan el rumbo. Los pronósticos permiten adecuar la producción a la razón de producción y al horizonte de planeación estableciendo los siguientes parámetros para ambos tipos de planes (cero inventarios y fuerza laboral constante): el primero es el costo de producción como guía en la toma de decisiones y el segundo es la determinación de la fuerza laboral necesaria para producir una cantidad de producción, este parámetro aplicado a ambos planes es el que posee el mayor peso para la toma de decisiones.

\section{Referencias}

[1] J. Domínguez Machuca, Á. G. M. M. Domínguez Machuca, S. García González y A. Ruiz Jiménez, Dirección de Operaciones. Aspectos tácticos y operativos en la producción y los servicios, McGraw-Hill, 1995.

[2] D. Sipper, Planeación y control de la producción, México D.F.: McGraw-Hill, 1998. 
[3] J. Raposo, A. Matos y A. Nunes, «Planning and Control of Production in the Improvement Manufacturing Process of Production Tables Games: A Case Study,» Book of Proceedings of the 7th International Conference on Industrial Engineering and Industrial Management, pp. 1060-1068, 2013.

[4] R. Carro, El sistema de producción y operaciones. Administración de las operaciones, 2014.

[5] M. ValenciA, F. Díaz y J. Correa, «Planeación de inventarios con demanda dinámica. Una revisión del estado del arte,» Dyna, vol. 190, n 82, pp. 183-191, 2015.

[6] R. del Solar, I. Chacón y M. Ponce, «Plan agregado de producción en barracas madereras. estudio de caso para una pequeña industria.,» Maderas: Ciencia y Tecnología, vol. 2, n 10, pp. 77-92, 2008.

[7] J. Rojas y S. Escudero, Reprogramación de operaciones en procesos de producción: Complejidad y alternativas de solución, México D.F.: Instituto Politécnico Nacional, 2000.

[8] A. Núñez, L. Guitart y X. Baraza, Dirección de operaciones: Decisiones tácticas y operativas, Barcelona : UOC, 2014.

[9] S. Narasimhan, Planeación de la producción y control de inventarios, México: Prentice Hall Hispanoamericana, 1996.

[10] O. Dante, A. Corominas, A. Lusa y O. Boiteux, Estado del arte sobre planificación agregada de la producción, Cataluña: Instituto de la organización y sistemas industriales: Barcelona, 2007.

[11] C. C. Holt, F. Modigliani, y H. A. Simon, «Linear decision rule for production and employment scheduling,» Management Science, vol. 2, pp. 1-30, 1955.

[12] C. C. Holt, F. Modigliani, J. F. Muth y H. A. Simon, «Derivation of a linear decision rule for production and employment,» Management Science,, vol. 2, pp. 159-177, 1956.

[13] C. C. Holt, F. Modigliani y J. F. Muth, «Planning production, inventory and workforce,» New York: Prentice Hall,, 1960.

[14] E. S. Buffa, Operations management; Problems and models., Jhon Wiley \& Sons., 1968.

[15] A. Hax, «Handbook of operations research. Models and applications,» Litton Educational Publishing ed, 1978.

[16] J. Mula, R. Poler y J. García, «Evaluación de Sistemas para la Planificación y Control de la Producción.,» Información tecnológica, vol. 1, n 17, pp. 19-34, 2006.

[17] A. Gunasekaran y H. Marri, «Application of aggregate production planning in developing countries," International Journal of Computer Applications in Technology, vol. 4, n 20, pp. 172-179, 2004.

[18] G. Buxey, «Aggregate planning for seasonal demand; reconciling theory with practice,» International Journal of Operations \& Production Management, vol. 11, n²5, pp. 1083-1100, 2005.

[19] T. Vollmann, Planeación y control de la producción, México: McGraw Hill, 2005.

[20] J. Cruelles Ruiz, Stock, Procesos y Dirección de Operaciones, Marcombo, 2015.

[21] R. Pindyck y D. Rubinfeld, Econometría, modelos y pronósticos, México: McGraw Hill, 2001.

[22] J. Heizer y B. Render, Dirección de la producción-Decisiones tácticas, Sexta ed., Editorial Prentice-Hall., 2001.

[23] S. Nahmias, Análisis de la producción y operaciones, McGraw Hill., 2014.

[24] J. Singhal y K. Singhal, Holt, Modigliani, Muth, and Simons work and its role in the renaissance and evolution of operations management, Journal of Operations Management, 2006.

[25] J. Acevedo y G. Mejía, Programación Reactiva y Robusta de la Producción en un Ambiente Sistema de Manufactura Flexible: Llegada de Nuevas Órdenes y Cambios en la Prioridad de las Órdenes del Trabajo, Bogotá, Colombia: Universidad de los Andes, 2006.

[26] J. Orlicky, Material Requirements Planning, Londres: McGraw Hill, 1975. 